\title{
AMPLITUDES E PADRÕES DE POLARIZAÇÃO DE PULSOS EM MEIOS ANISOTRÓPICOS
}

\author{
Rubenvaldo Monteiro Pereira ${ }^{1}$, João dos Santos Protázio² e Jessé Carvalho Costa ${ }^{3}$ \\ Recebido em 21 novembro, 2008 / Aceito em 17 abril, 2009 \\ Received on November 21, 2008 / Accepted on April 17, 2009
}

\begin{abstract}
Extracting subsurface lithological information from seismic data has become a great challenge to seismic exploration, as the hypotheses of stratification made by isotropic layers has been insufficient to represent the behavior of elastic field in surveys with great offset between source and receiver, multicomponent geophones, three-dimensional Vertical Seismic Profile (VSP) measures and so forth. Due to this reason, a more realistic model of subsurface is demanded. As a result, the seismic prospecting starts to consider the anisotropic models of subsurface to characterize reservoirs, for example. This paper aims at showing formalism to model the scattering of pulses from incident plane waves in horizontal plain interfaces which separate anisotropic media. This scattering is obtained primarily through the explicit formulation of traction and deformation fields as a function of propagator, polarization and impedance matrices of media. After that, this formalism is used to obtain the reflection and transmission coefficients matrices through a horizontal plain interface to be, subsequently generalized to a scattering through multiple layers. Finally, the amplitude of an analytical pulse is inserted in the incident wave to calculate the scattering of the pulse through stratifications.
\end{abstract}

Keywords: anisotropy, plane wave, scattered, pulses.

RESUMO. Extrair informações litológicas da subsuperfície através de dados sísmicos constitui-se num grande desafio à prospecção sísmica, pois a hipótese de estratificações formadas por camadas isotrópicas se mostra insuficiente para representar o comportamento do campo elástico em levantamentos com grandes afastamentos entre fonte e receptor, geofones multicomponentes, medidas de VSP tridimensional, entre outros. Sob este panorama, a prospecção sísmica passa a considerar modelos anisotrópicos de subsuperfície para, por exemplo, caracterizar reservatórios. 0 objetivo deste texto é apresentar um formalismo para modelar o espalhamento de pulsos a partir de ondas planas incidentes em interfaces planas horizontais que separam meios anisotrópicos. Este espalhamento é obtido primeiramente, através da formulação explícita dos campos de deformação e tração como função das matrizes propagadoras, de polarização e de impedância do meio. Em seguida este formalismo é usado para a obtenção das matrizes dos coeficientes de reflexão e transmissão através de uma interface plana horizontal para posteriormente, ser generalizado para o espalhamento através de múltiplas camadas. Finalmente, inserem-se ao campo da onda incidente as amplitudes de um pulso analítico para calcular 0 espalhamento do pulso através de estratificações.

Palavras-chave: anisotropia, ondas planas, espalhamento, pulsos.

\footnotetext{
1 Universidade Federal do Pará - UFPa/Campus Tocantins Cametá, Faculdade de Matemática, Trav. Padre Antônio Franco s/n, Matinha, 68400-000 Cametá, PA, Brasil. Tel./Fax: (91) 3781-1182 - E-mail: rubenp@ufpa.br

2Universidade Federal do Pará - UFPa, Faculdade de Estatística, Trav. Augusto Correa, 1, Guamá, 66075-900 Belém, PA, Brasil. Tel./Fax: (91) 3201-7413 -E-mail: protazio@ufpa.br

3 Universidade Federal do Pará - UFPa, Programa de Pós-Graduação em Geofísica, Trav. Augusto Correa, 1, Guamá, 66075-900 Belém, PA, Brasil. Tel./Fax: (91) 3201-7693 - E-mail: jesse@ufpa.br
} 


\section{INTRODUÇÃo}

A formulação de um modelo analítico para o estudo da propagação de pulsos através de interfaces planas que separam meios elásticos anisotrópicos é o objetivo principal deste trabalho.

A necessidade de se estudar modelos anisotrópicos, no estudo da propagação de ondas através de meios elásticos, para a prospecção geofísica, advém do surgimento de novas técnicas de aquisição de dados, tais como: as medidas de Perfil Sísmico Vertical (PSV) tridimensional, os levantamentos com grandes afastamentos entre fonte e receptor; além de levantamentos com geofones multicomponentes, entre outras.

Por outro lado, este trabalho utiliza como modelos de subsuperfície, nos experimentos numéricos, meios anisotrópicos induzidos por fraturas segundo o modelo de Hudson (1981), pelo fato da presença de fraturas naturais em reservatórios constituir um fator importante na determinação do fluxo de fluidos durante a produção e, também, por estes meios comportarem-se efetivamente como meios anisotrópicos.

Sismogramas sintéticos completos de ondas planas, construídos com as expressões analíticas das auto-soluções, já foram estudados por Fryer \& Frazer (1987), e posteriormente adaptado para experimentos em Perfil Sísmico Vertical (PSV) por Mallick \& Frazer (1988), considerando, nestes casos, meios anisotrópicos estratificados com pelo menos um plano de simetria especular. Já Crampin (1985) usou, além de sismogramas sintéticos, hodogramas (PSV) das ondas cisalhantes para analisar o fenômeno da birrefringência presente em meios anisotrópicos.

Um estudo detalhado da reflexão e da transmissão de pulsos em meios anisotrópicos foi feito por Protázio (1994), que considerou 0 espalhamento de ondas compressionais $P$ e cisalhantes $(S$ e $T$ ) e suas respectivas múltiplas, obtido a partir de uma onda incidente $P$ em meios isotrópicos e anisotrópicos com pelo menos um plano de simetria especular. 0 objetivo principal deste trabalho foi marcar o comportamento pós-crítico do espalhamento destes pulsos.

Neste texto é feita uma extensão do trabalho elaborado por Protázio (1994) ao considerar um modelo matemático explícito para a propagação de pulsos em meios anisotrópicos.

0 modelo é robusto para estudar reflexão e transmissão de ondas $P, S$ e $T$, porém os experimentos numéricos apresentados aqui tratam apenas do caso de ondas incidentes $P$ e de suas refletidas e transmitidas $P$. Os resultados obtidos ratificam resultados obtidos nos trabalhos de Protázio (1994) e Gomes (1999) e nos abre novas perspectivas de estudo.

\section{METODOLOGIA \\ Fundamentos teóricos}

Em um meio perfeitamente elástico, linear, homogêneo e anisotrópico, com densidade $\rho$ e parâmetros elásticos $c_{i j k l}$, a equação geral da onda é dada por:

$$
\rho \partial_{t t} w_{i}=\partial_{j} \tau_{i j}+f_{i},
$$

sendo $w_{i} 0$ campo de onda; $\partial_{t t}$ a segunda derivada no tempo; $\partial_{j}$ a $j$-ésima derivada espacial e $f_{i}$ uma fonte externa associada ao problema.

Em regime de deformação infinitesimal vale a Lei de Hooke generalizada dada por:

$$
\tau_{i j}=c_{i j k l} \partial_{l} w_{k} .
$$

Assim sendo, a Equação (1) passa a ser representada por:

$$
\rho \partial_{t t} w_{i}=\partial_{j}\left(c_{i j k l} \partial_{l} w_{k}\right)+f_{i} .
$$

Considere-se agora uma onda harmônica plana (Aki \& Richard, 1980):

$$
w_{i}=A n_{j} e^{-i \omega\left(t-x_{k} s_{k}\right)}
$$

sendo $A$ a amplitude da onda; $n_{j}$ a sua polarização unitária; $t 0$ tempo; $\omega$ a frequência e $s_{k} 0$ vetor de vagarosidade, que determina a direção normal da frente de onda. A substituição da Equação (4) na Equação (3), tomando-se $f_{i}=0$, gera a conhecida equação de Christoffel:

$$
\left(\Gamma_{i k}-\rho \delta_{i k}\right) n_{k}=0 \quad \text { ou } \quad\left(\tilde{\Gamma}_{i k}-v^{2} \delta_{i k}\right) n_{k}=0,
$$

sendo $\Gamma_{i k}=c_{i j k l} s_{i} s_{j}$ ou $\tilde{\Gamma}_{i k}=a_{i j k l} m_{l} m_{j}$ a chamada matriz de Christoffel, a qual é simétrica e positiva definida. Aqui, $a_{i j k l}=c_{i j k l} / \rho$ e $m_{i}$ é um vetor unitário na direção da vagarosidade $s_{k}=m_{k} / v$, sendo $v$ a velocidade de fase e $\delta_{i j}$ 0 conhecido delta de Krönecker definido por: $\delta_{i j}=1$, se $i=j$ e $\delta_{i j}=0$ se $i \neq j$. Para meios sem atenuação, os coeficientes da matriz de Christoffel são reais e 0 sistema linear homogêneo da Equação (5) constitui um problema de autovalor real. Soluções não-triviais da Equação (5) exigem a condição:

$$
\operatorname{det}\left(a_{i j k l} m_{l} m_{j}-v^{2} \delta_{i j}\right)=0
$$

chamado de polinômio de Christoffel. Portanto, para uma dada direção unitária $m_{i}$, a Equaçã̃o (6) produz três velocidades de fase $v$, em geral distintas, que podem ser ordenadas como $v_{T} \leq$ $v_{S} \leq v_{P}$, com os subíndices $P, S$ e $T$ representando as ondas com maior, média e menor velocidade de fase, respectivamente. As velocidades dependem das mesmas variáveis $a_{i j k l}$ 
e $m_{i}$. A dependência de $m_{i}$ implica na dependência da velocidade de fase com relação à direção de propagação da onda. Assim para cada velocidade $v_{Q}, Q=P, S$ e $T$ na Equação (6) haverá um correspondente autovetor real unitário $n_{I Q}$, conhecido como a polarização associada. Se todas as três velocidades são diferentes, as respectivas polarizações ficam determinadas ao longo de três direções mutuamente ortogonais. Assim, as três diferentes propagações de onda em um meio anisotrópico não diferem apenas pelas suas velocidades de fase $v_{Q}$, mas também pela orientação de suas polarizações $n_{I Q}$, chamada de polarização linear, que fisicamente especificam as direções de deslocamento da partícula $w_{i}$.

\section{0 espalhamento de ondas planas através de meios anisotrópicos}

Considere-se, agora, um meio elástico anisotrópico arbitrário, com 0 campo de uma onda plana em um horizonte arbitrário $x_{3}$ descrito pela Equação (4) e orientado por um sistema de referência cartesiano tridimensional. Assim, para cada componente horizontal $s_{H}=\left(s_{1}, s_{2}\right)^{t}$ fixa (Lei de Snell), a Equação (6) é de sexto grau em $s_{3}$ definindo uma superfície no espaço $s$ que determina os possíveis valores da vagarosidade como função da direção de propagação e cujas raízes podem ser reais ou complexas, no caso da ocorrência de eventos pré ou póscríticos, respectivamente. No caso das raízes reais, estes eventos podem, ainda, ser classificados como descendentes (denotado com um sobre-índice $D$ ) ou ascendentes (denotado com um sobre-índice $U$ ) conforme a componente vertical da velocidade de grupo associada seja positiva ou negativa. Já em eventos pós-críticos, e considerando-se 0 fato dos coeficientes da equação característica serem reais, as raízes complexas aparecem em pares conjugados, podendo-se escolher as componentes de $s_{3}$ como sendo descendente ou ascendente conforme estas atenuem a amplitude com a profundidade. Segue das condições de radiação que para $\omega>0: \operatorname{Im}\left(s_{3}^{D}\right)>0$ e $\operatorname{Im}\left(s_{3}^{U}\right)<0$, sendo $s_{3}^{D}$ e $s_{3}^{U}$ os eventos descendente e ascendente, respectivamente. Assim, as seis componentes $s_{3}$ são separadas em dois conjuntos de três componentes cada: um ascendente $\left\{\mathbf{s}_{3 P}^{U}, \mathbf{s}_{3 S}^{U}, \mathbf{s}_{3 T}^{U}\right\}$, e outro descendente, $\left\{\mathbf{s}_{3 P}^{D}, \mathbf{s}_{3 S}^{D}, \mathbf{s}_{3 T}^{D}\right\}$. Por outro lado, as polarizações também vão ficar divididas em dois conjuntos: um ascendente, $\left\{\mathbf{n}_{P}^{U}, \mathbf{n}_{S}^{U}, \mathbf{n}_{T}^{U}\right\}$ e outro descendente, $\left\{\mathbf{n}_{P}^{D}, \mathbf{n}_{S}^{D}, \mathbf{n}_{T}^{D}\right\}$. Uma representação esquemática destes efeitos pode ser observada nas Figuras 1a e 1b, nas quais podemos visualizar duas superfícies de vagarosidade no plano $x y$, sendo que na Figura 1a com plano de simetria especular horizontal e na Figura 1b sem esta simetria.
Agora, superpondo-se os efeitos de todos os eventos ascendentes e descentes em cada horizonte $x_{3}, 0$ campo de onda total é dado por:

$$
\begin{aligned}
\mathbf{w}= & e^{-i \omega\left(t-\left\langle\mathbf{s}_{H}, \mathbf{x}_{H}\right\rangle\right)} \mathbf{N}^{D} \mathbf{e}^{i \omega x_{3} \mathbf{s}_{3}^{D}} \mathbf{d} \\
& +e^{-i \omega\left(t-\left\langle\mathbf{s}_{H}, \mathbf{x}_{H}\right\rangle\right)} \mathbf{N}^{U} \mathbf{e}^{i \omega x_{3} \mathbf{s}_{3}^{U}} \mathbf{u},
\end{aligned}
$$

sendo $\mathbf{d} \mathrm{e} \mathbf{u}$ vetores em que as componentes são as amplitudes das ondas descendentes e ascendentes, respectivamente e \langle\rangle 0 produto interno de vetores. As matrizes de polarização $\mathbf{N}^{D}$ e $\mathbf{N}^{U}$ guardam em suas colunas as informações das polarizações das ondas ascendentes e descendentes, respectivamente e apresentam as formas:

$$
\mathbf{N}^{D}=\left[\mathbf{n}_{P}^{D} \mathbf{n}_{S}^{D} \mathbf{n}_{T}^{D}\right] \text { e } \mathbf{N}^{U}=\left[\mathbf{n}_{P}^{U} \mathbf{n}_{T}^{U} \mathbf{n}_{T}^{U}\right]
$$

enquanto as matrizes diagonais $\mathbf{S}_{3}^{D}$ e $\mathbf{S}_{3}^{U}$ guardam as informações das componentes verticais da vagarosidade das ondas ascendentes e descendentes, respectivamente e apresentam as formas:

$$
\mathbf{S}_{3}^{D}=\operatorname{diag}\left[\mathbf{s}_{3 P}^{D} \mathbf{s}_{3 S}^{D} \mathbf{s}_{3 T}^{D}\right] \text { e } \mathbf{S}_{3}^{U}=\operatorname{diag}\left[\mathbf{s}_{3 P}^{U} \mathbf{s}_{3 S}^{U} \mathbf{s}_{3 T}^{U}\right] .
$$

Por outro lado, o campo vertical de tração apresenta a forma:

$$
\begin{aligned}
\tau_{3}= & e^{-i \omega\left(t-\left\langle\mathbf{s}_{H}, \mathbf{x}_{H}\right\rangle\right)} \mathbf{Z}^{D} \mathbf{e}^{i \omega x_{3} \mathbf{s}_{3}^{D}} \mathbf{d} \\
& +e^{-i \omega\left(t-\left\langle\mathbf{s}_{H}, \mathbf{x}_{H}\right\rangle\right)} \mathbf{Z}^{U} \mathbf{e}^{i \omega x_{3} \mathbf{s}_{3}^{U}} \mathbf{u},
\end{aligned}
$$

sendo $\mathbf{Z}^{D}$ e $\mathbf{Z}^{U}$ as matrizes de impedância do meio, descendente e ascendente, respectivamente (Gomes, 1999).

Como em cada horizonte $x_{3}$ os campos $\mathbf{w}$ e $\tau_{3}$ são considerados, por hipótese, contínuos pode-se definir o campo como:

$$
\mathbf{b}\left(x_{3}\right)=\left[\begin{array}{c}
\mathbf{w} \\
\tau_{3}
\end{array}\right]=e^{-i \omega\left(t-\left\langle\mathbf{s}_{H}, \mathbf{x}_{H}\right\rangle\right)} \mathbf{N e}^{i \omega x_{3} \mathbf{s}_{3}} \mathbf{v},
$$

sendo

$$
\begin{aligned}
& \mathbf{N}=\left[\begin{array}{ll}
\mathbf{N}^{D} & \mathbf{N}^{U} \\
\mathbf{Z}^{D} & \mathbf{Z}^{U}
\end{array}\right], \mathbf{v}=\left[\begin{array}{l}
\mathbf{d} \\
\mathbf{u}
\end{array}\right] \mathrm{e} \\
& \mathbf{e}^{i \omega x_{3} \mathbf{s}_{3}}=\left[\begin{array}{cc}
\mathbf{e}^{i \omega x_{3} \mathbf{s}_{3}^{D}} & 0 \\
0 & \mathbf{e}^{i \omega x_{3} \mathbf{s}_{3}^{U}}
\end{array}\right] .
\end{aligned}
$$

\section{0 espalhamento através de interface plana horizontal}

A representação obtida no tópico anterior é adequada para a obtenção do espalhamento de ondas através de uma interface plana horizontal, separando dois meios anisotrópicos. Para isto, faz-se $x_{3}=0$ e considera-se a onda incidente no semiespaço superior $\left(x_{3}<0\right)$ e a onda transmitida no semiespaço inferior 
(a)

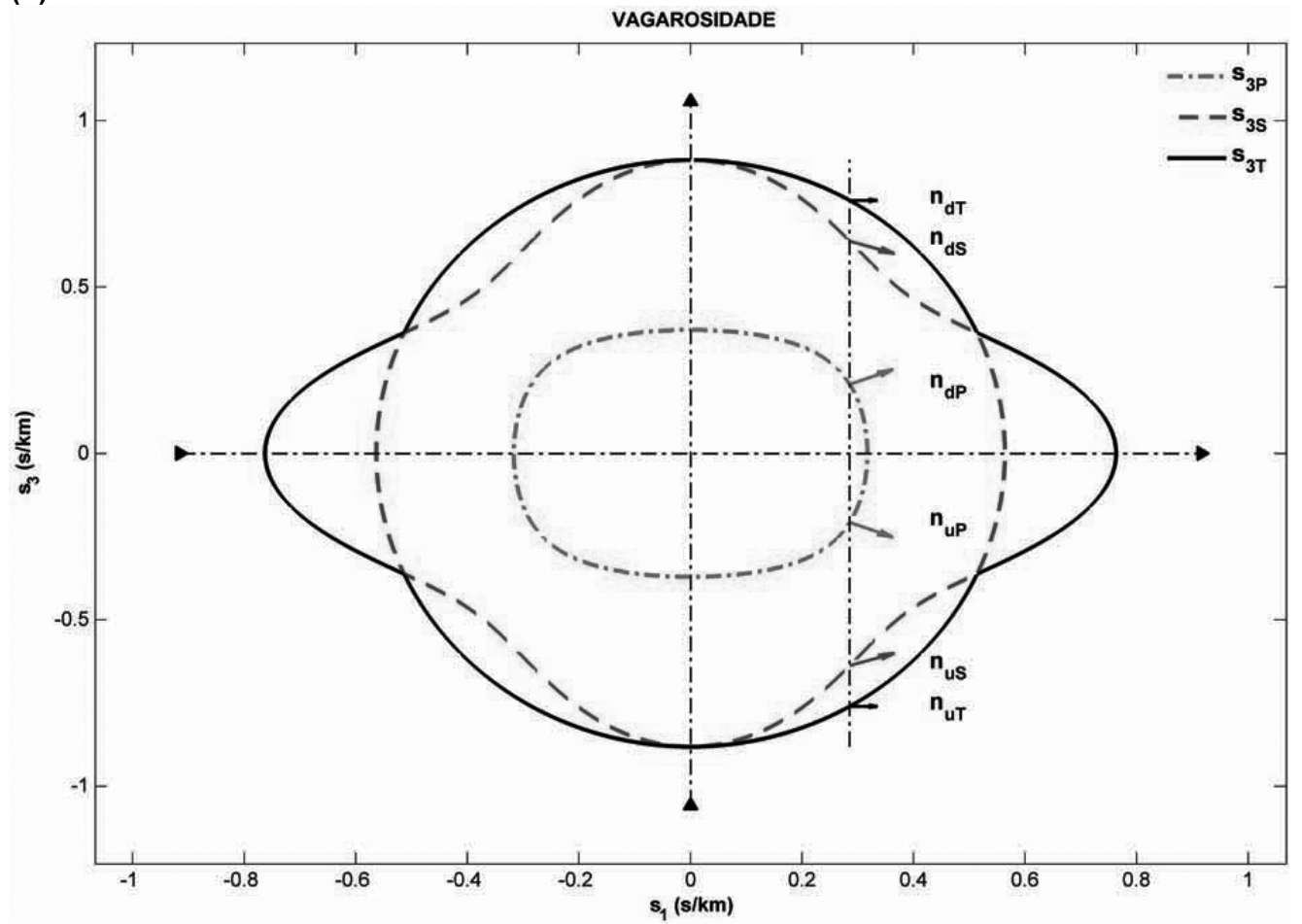

(b)

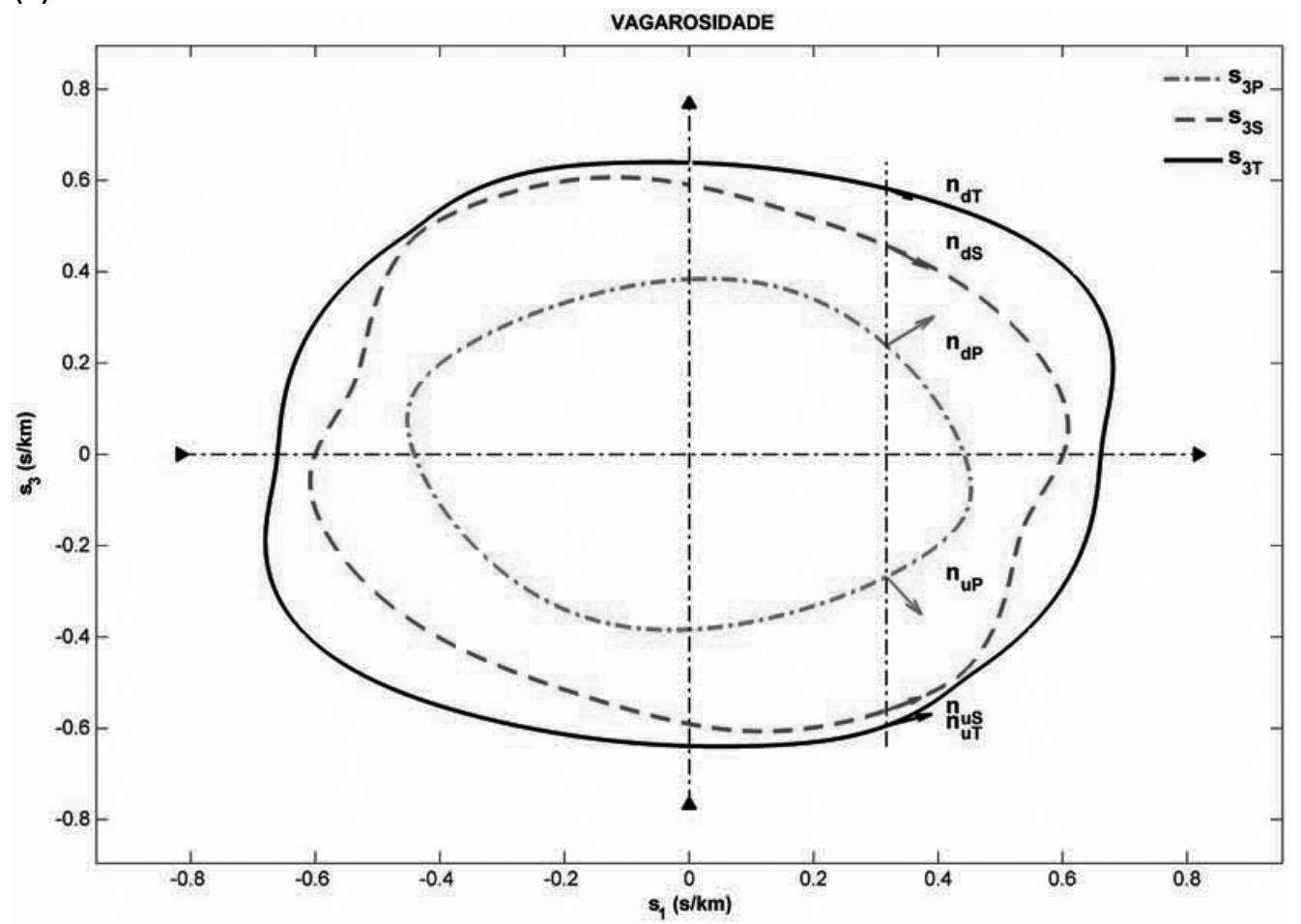

Figura 1 - (a) Superfície de vagarosidade em um plano de simetria para um meio ortorrômbico. Cada vagarosidade horizontal $\mathbf{S}_{H}$ determina seis componentes verticais: três ascendentes $\left\{\mathbf{S}_{3 P}^{U}, \mathbf{S}_{3 S}^{U}, \mathbf{S}_{3 T}^{U}\right\}$ e três descendentes $\left\{-\mathbf{S}_{3 P}^{U},-\mathbf{S}_{3 S}^{U},-\mathbf{S}_{3 T}^{U}\right\}$ observando-se a simetria horizontal. As direções de polarização também são apresentadas. (b) Superfície de vagarosidade em um plano sagital para um meio triclínico. Cada vagarosidade horizontal $\mathbf{S}_{H}$ determina seis componentes verticais: três ascendentes $\left\{\mathbf{s}_{3 P}^{U}, \mathbf{s}_{3 S}^{U}, \mathbf{s}_{3 T}^{U}\right\}$ e três descendentes $\left\{\mathbf{S}_{3 P}^{D}, \mathbf{S}_{3 S}^{D}, \mathbf{S}_{3 T}^{D}\right\}$ observando-se a ausência de simetria horizontal. As direções de polarização também são apresentadas. 
$\left(x_{3}>0\right)$ conforme mostrado na Figura 2. No semiespaço superior ocorrem a incidência e a reflexão das amplitudes $\mathbf{i}$ er respectivamente, com as matrizes de polarização incidente e refletida representadas por $\mathbf{N}_{i}$ e $\mathbf{N}_{r}$, e as matrizes de impedância incidente e refletida por $\mathbf{Z}_{i}$ e $\mathbf{Z}_{r}$ respectivamente. No meio inferior, acontece a transmissão das ondas com amplitude $\mathbf{t}$, com matrizes de polarização $\mathbf{N}_{t}$ e matrizes de impedância $\mathbf{Z}_{t}$ respectivamente.

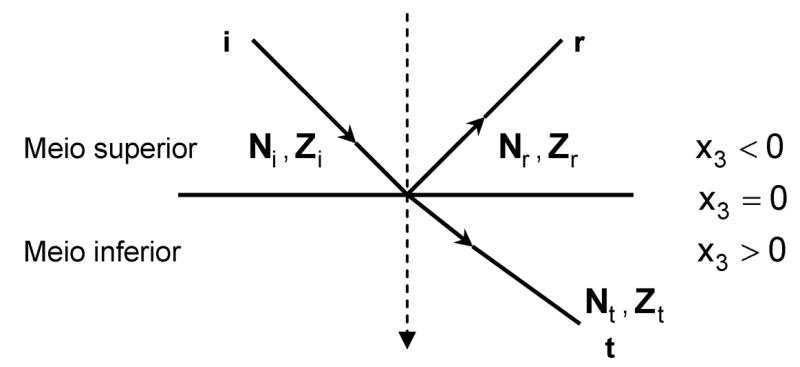

Figura 2 - Modelo esquemático do espalhamento de ondas em interface plana horizontal. Observam-se as amplitudes das ondas, as matrizes de impedância e de polarização incidente, refletida e transmitida, envolvidas no espalhamento da onda.

Considerando-se a continuidade do campo $\mathbf{b}\left(x_{3}\right)$ em $x_{3}=$ 0 , obtém-se a identidade:

$$
\left[\begin{array}{ll}
\mathbf{N}_{i} & \mathbf{N}_{r} \\
\mathbf{Z}_{i} & \mathbf{Z}_{r}
\end{array}\right]\left[\begin{array}{l}
\mathbf{i} \\
\mathbf{r}
\end{array}\right]=\left[\begin{array}{ll}
\mathbf{N}_{t} & \mathbf{0} \\
\mathbf{Z}_{t} & \mathbf{0}
\end{array}\right]\left[\begin{array}{l}
\mathbf{t} \\
\mathbf{0}
\end{array}\right],
$$

que é o sistema de equações em representação matricial, onde as equações são:

$$
\left\{\begin{array}{l}
\mathbf{N}_{i} \mathbf{i}+\mathbf{N}_{r} \mathbf{r}=\mathbf{N}_{t} \mathbf{t} \\
\mathbf{Z}_{i} \mathbf{i}+\mathbf{Z}_{r} \mathbf{r}=\mathbf{Z}_{t} \mathbf{t}
\end{array}\right.
$$

Considerando-se, por hipótese, a inversibilidade das matrizes envolvidas, uma possível solução explíita do sistema na Equação (13) é dada por (Gomes, 1999)

$$
\begin{aligned}
\mathbf{t} & =\left(\mathbf{Z}_{t}-\mathbf{Z}_{r} \mathbf{N}_{r}^{-1} \mathbf{N}_{t}\right)^{-1}\left(\mathbf{Z}_{i}-\mathbf{Z}_{r} \mathbf{N}_{r}^{-1} \mathbf{N}_{i}\right) \\
\mathbf{r} & =\mathbf{N}_{r}^{-1}\left(\mathbf{N}_{t} \mathbf{t}-\mathbf{N}_{i} \mathbf{i}\right) .
\end{aligned}
$$

Caso não seja possível inverter as matrizes envolvidas tem-se a presença de ondas de Stonley.

\section{O espalhamento de ondas através de multicamadas}

Fryer \& Frazer (1987) apresentam um método matricial para análise de ondas planas em meios homogêneos, considerando a equação do movimento (Eq. (1)) e as relações constitutivas (Eq. (2)). Neste formalismo, as derivadas de primeira ordem com relação à profundidade da tensão e deslocamento são necessárias e 0 estudo do espalhamento fica reduzido à solução do sistema de equações diferenciais:

$$
\frac{d \mathbf{b}}{d x_{3}}=\mathbf{Q} \mathbf{b},
$$

sendo $\mathbf{Q}$ uma matriz constante, com entradas dependendo das propriedades elásticas do meio, da vagarosidade horizontal e da frequência.

Derivando-se a equação do campo (Eq. (11)) em relação ao horizonte $x_{3}$, obtém-se:

$$
\frac{d \mathbf{b}}{d x_{3}}=i \omega e^{-i \omega\left(t-\left\langle\mathbf{s}_{H}, \mathbf{x}_{H}\right\rangle\right)} \mathbf{N S}_{3} \mathbf{e}^{i \omega x_{3} \mathbf{S}_{3}} \mathbf{v},
$$

mostrando que o campo b( $\left.x_{3}\right)$ satisfaz (Pereira, 2003):

$$
\frac{d \mathbf{b}}{d x_{3}}=i \omega\left(\mathbf{N S}_{3} \mathbf{N}^{-1}\right) \mathbf{b} .
$$

A sua solução formal é:

$$
\mathbf{b}\left(x_{3}\right)=\mathbf{e}^{i \omega\left(\mathbf{N S}_{3} \mathbf{N}^{-1}\right)\left(x_{3}-x_{30}\right)} \mathbf{b}\left(x_{30}\right),
$$

sendo $x_{30}$ e $x_{3}$ as coordenadas na base e no topo, respectivamente, de uma camada de espessura $h=x_{3}-x_{30}$. A representação obtida acima pode ser reescrita como:

$$
\mathbf{b}\left(x_{3}\right)=\mathbf{N} \mathbf{e}^{i \omega h \mathbf{S}_{3}} \mathbf{N}^{-1} \mathbf{b}\left(x_{30}\right),
$$

mostrando que

$$
\mathbf{Q}(h)=\mathbf{N} \mathbf{e}^{i \omega h \mathbf{S}_{3}} \mathbf{N}^{-1}
$$

corresponde exatamente à matriz de propagação através da camada, estudada por Woodhouse (1974) uma vez que ela permite a determinação do campo de onda na base a partir do campo de onda no topo.

No caso particular de propagação em regime quase estático, ou seja, em que $\left\|\omega h \mathbf{S}_{3}\right\| \ll 1$, a matriz de propagação pode ser aproximada por:

$$
\mathbf{Q}(h)=\mathbf{N} \mathbf{e}^{i \omega h \mathbf{S}_{3}} \mathbf{N}^{-1} \approx \mathbf{I}+i \omega h \mathbf{N} \mathbf{S}_{3} \mathbf{N}^{-1} .
$$

\section{O espalhamento de pulsos através de meios anisotrópicos}

Segundo Protázio (1994), para cada frequência $\omega$, 0 campo de onda incidente apresenta a forma:

$$
\overline{\mathbf{w}}_{i}(\omega)=\mathbf{N}_{i} \mathbf{e}^{i \omega x_{3} \mathbf{S}_{3 i}} \mathbf{i}(\omega),
$$

sendo $\tau=t-\left\langle\mathbf{s}_{H}, \mathbf{x}_{H}\right\rangle, \mathbf{S}_{3 i}$ a matriz das componentes verticais das vagarosidades incidentes e $\mathbf{i}(\omega)=\left[F_{P}(\omega)\right.$, $\left.F_{P}(\omega), F_{P}(\omega)\right]^{T} 0$ vetor das amplitudes das ondas incidentes no domínio das frequências. Aplicando-se a transformada 
de Fourier, o campo incidente pode ser expresso, no domínio do tempo, como:

$$
\begin{aligned}
\mathbf{w}_{i}(t) & =\frac{1}{2 \pi} \int_{-\infty}^{+\infty} e^{-i \omega \tau} \mathbf{N}_{i} \mathbf{e}^{i \omega x_{3} \mathbf{S}_{3 i} \mathbf{i}(\omega) d \omega,} \\
& =\left[\begin{array}{lll}
n_{1 P i} & n_{1 S i} & n_{1 T i} \\
n_{2 P i} & n_{2 S i} & n_{2 T i} \\
n_{3 P i} & n_{3 S i} & n_{3 T i}
\end{array}\right]\left[\begin{array}{c}
f_{P}\left(\tau-S_{3 P i} X_{3}\right) \\
f_{S}\left(\tau-S_{3 S i} X_{3}\right) \\
f_{T}\left(\tau-S_{3 T i} X_{3}\right)
\end{array}\right],
\end{aligned}
$$

sendo $f_{Q}(t)$ a assinatura da fonte no tempo.

De forma análoga, o campo refletido, no domínio do tempo, é dado por:

$$
\mathbf{w}_{r}(t)=\frac{1}{2 \pi} \int_{-\infty}^{+\infty} e^{-i \omega \tau} \mathbf{N}_{r} \mathrm{e}^{i \omega x_{3} \mathbf{S}_{3 r}} \mathbf{R}(\omega) \mathbf{i}(\omega) d \omega,
$$

sendo $\mathbf{S}_{3 R}$ a matriz das componentes verticais das vagarosidades refletidas e o campo transmitido por:

$$
\mathbf{w}_{t}(t)=\frac{1}{2 \pi} \int_{-\infty}^{+\infty} e^{-i \omega \tau} \mathbf{N}_{t} \mathbf{e}^{i \omega x_{3} \mathbf{S}_{3 t}} \mathbf{T}(\omega) \mathbf{i}(\omega) d \omega,
$$

sendo $\mathbf{S}_{3 t}$ a matriz das componentes verticais das vagarosidades transmitidas.

\section{O sinal analítico dos pulsos espalhados}

Ainda segundo Protázio (1994), dado um sinal temporal $f(\tau), 0$ sinal analítico a ele associado tem a forma $f^{(A)}(\tau)=f(\tau)+$ $i f_{H}(\tau)$ sendo $f_{H}(\tau)$ a transformada de Hilbert do sinal, definida por:

$$
f_{H}(\tau)=\int_{-\infty}^{+\infty} \frac{f(t)}{t-\tau} d \tau
$$

Para a obtenção do sinal analítico do pulso refletido, considera-se aqui apenas 0 caso pós-crítico, pois todo o processo de espalhamento das ondas no caso pré-crítico é real. Assim o sinal analítico refletido através de uma interface, na sua forma mais geral, é dado por:

$$
\begin{aligned}
& \mathbf{w}_{r}(t)=\frac{1}{\pi} \mathbf{N}^{U} \mathbf{R} \int_{-\infty}^{+\infty} \\
& \times e^{-i \omega \tau} \mathbf{e}^{i \omega x_{3} \operatorname{Re}\left(\mathbf{S}_{3}^{U}\right)} \mathbf{e}^{\omega x_{3}}\left|\operatorname{Im}\left(\mathbf{S}_{3}^{U}\right)\right| \mathbf{i}(\omega) d \omega,
\end{aligned}
$$

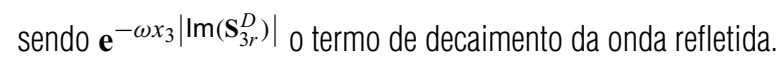

Já o pulso analítico transmitido na sua forma mais geral é dado por:

$$
\begin{aligned}
& \mathbf{w}_{t}(\tau)=\frac{1}{\tau} \mathbf{N}^{D} \mathbf{T} \int_{-\infty}^{+\infty} \\
& \times e^{-i \omega \tau} \mathbf{e}^{i \omega x_{3} \operatorname{Re}\left(\mathbf{S}_{3}^{D}\right)} \mathbf{e}^{-\omega x_{3}\left|\operatorname{Im}\left(\mathbf{S}_{3}^{D}\right)\right|} \mathbf{i}(\omega) d \omega,
\end{aligned}
$$

sendo $\mathbf{e}^{-\omega x_{3}\left|\operatorname{Im}\left(S_{3 r}^{D}\right)\right|} 0$ termo de decaimento da onda transmitida.

\section{0 espalhamento de pulsos através de estratificações}

Considerando-se, agora, uma estratificação com $N$ camadas, limitada por um semiespaço superior e por um semiespaço inferior e seguindo o método iterativo, a matriz de propagação relacionando às interfaces superior e inferior é dada pelo produto das matrizes de propagação de cada camada (Protázio, 1994). Assim, o campo na base é dado por:

$$
\mathbf{b}\left(x_{3 b}\right)=\prod_{k=N}^{1} \mathbf{Q}_{k}\left(h_{k}\right) \mathbf{b}\left(x_{3 t}\right) \equiv \mathbf{Q} \mathbf{b}\left(x_{3 t}\right)
$$

sendo $\mathbf{Q}_{k}\left(h_{k}\right)$ a matriz de propagação calculada em uma $k$ ésima camada de uma estratificação com espessura $h_{k}$. Protázio (1994) mostrou que:

$$
\begin{aligned}
{\left[\begin{array}{l}
\mathbf{t} \\
\mathbf{0}
\end{array}\right] } & =\mathbf{E}_{N+1, N} \mathbf{P}_{N} \mathbf{E}_{N, N-1} \cdots \mathbf{E}_{2,1} \mathbf{P}_{1} \mathbf{E}_{1,0}\left[\begin{array}{l}
\mathbf{i} \\
\mathbf{r}
\end{array}\right] \\
& =\mathbf{E}^{D}\left[\begin{array}{l}
\mathbf{i} \\
\mathbf{r}
\end{array}\right]=\left[\begin{array}{ll}
\mathbf{E}_{11}^{D} & \mathbf{E}_{12}^{D} \\
\mathbf{E}_{21}^{D} & \mathbf{E}_{22}^{D}
\end{array}\right]\left[\begin{array}{l}
\mathbf{i} \\
\mathbf{r}
\end{array}\right]
\end{aligned}
$$

sendo que cada $\mathbf{E}_{k, k-1}, k=1,2,3, \ldots, N+1$ representa uma matriz que controla 0 espalhamento na interface que separa as camadas $k$ e $k-1$ e cada $\mathbf{P}_{k}=k=1,2,3, \ldots, N$ corresponde à matriz de propagação através da $k$-ésima camada. $\mathrm{A}$ matriz $\mathbf{E}^{D}$ é denominada matriz descendente de espalhamento associada à estratificação, pois determina 0 espalhamento das ondas na interface inferior a partir do espalhamento na interface superior. A Equação (29) pode ser reescrita gerando:

$$
\left[\begin{array}{l}
\mathbf{i} \\
\mathbf{r}
\end{array}\right]=\left[\begin{array}{ll}
\mathbf{E}_{11}^{U} & \mathbf{E}_{12}^{U} \\
\mathbf{E}_{21}^{U} & \mathbf{E}_{22}^{U}
\end{array}\right]\left[\begin{array}{l}
\mathbf{t} \\
\mathbf{0}
\end{array}\right]
$$

onde $\mathbf{E}^{U}=\left(\mathbf{E}^{D}\right)^{-1}$ corresponde à matriz ascendente de espaIhamento associada à estratificação, pois determina o espalhamento das ondas na interface superior a partir do espalhamento na interface inferior. Assim, as soluções para os coeficientes $\mathbf{r}$ e $\mathbf{t}$ são obtidos e dados por

$$
\begin{aligned}
& \mathbf{r}=\mathbf{E}_{21}^{U}\left(\mathbf{E}_{11}^{U}\right)^{-1} \mathbf{i} \\
& \mathbf{t}=\left(\mathbf{E}_{11}^{U}\right)^{-1} \mathbf{i} .
\end{aligned}
$$

Para 0 caso de uma camada simples, a matriz ascendente de espalhamento é dada por $\mathbf{E}^{U}=\mathbf{E}_{0,1} \mathbf{P}_{1}^{-1} \mathbf{E}_{1,2}$, obtendo assim 
0 coeficiente de transmissão como:

$$
\begin{aligned}
\mathbf{t} & =\mathbf{T}_{12}\left(\mathbf{I}-\boldsymbol{\Lambda}_{1}^{D} \mathbf{R}_{10} \boldsymbol{\Lambda}_{1}^{U} \mathbf{R}_{12}\right)^{-1} \boldsymbol{\Lambda}_{1}^{D} \mathbf{T}_{01} \mathbf{i} \\
& \equiv \mathbf{T}_{12}(\mathbf{I}-\boldsymbol{\Pi})^{-1} \boldsymbol{\Lambda}_{1}^{D} \mathbf{T}_{01} \mathbf{i},
\end{aligned}
$$

sendo que os símbolos $\mathbf{R}_{j k}$ e $\mathbf{T}_{j k}$ representam as matrizes de reflexão e a transmissão da onda através da interface que separa os meios $j$ e $k$, no meio $k$. Em geral estas matrizes são $3 \times 3$ e tem a forma:

$$
\mathbf{R}=\left[\begin{array}{lll}
r_{P P} & r_{S P} & r_{T P} \\
r_{P S} & r_{S S} & r_{T S} \\
r_{P T} & r_{S T} & r_{T T}
\end{array}\right] \text { e } \mathbf{T}=\left[\begin{array}{ccc}
t_{P P} & t_{S P} & t_{T P} \\
t_{P S} & t_{S S} & t_{T S} \\
t_{P T} & t_{S T} & t_{T T}
\end{array}\right]
$$

Aqui, por exemplo, $r_{S P}$ é o coeficiente de reflexão de uma onda $S$ convertida de uma onda descendente incidente $P$. Cada matriz $\boldsymbol{\Lambda}_{j}^{D}=\mathbf{e}^{i \omega h_{j} \mathbf{S}_{3}^{D}}$ e $\boldsymbol{\Lambda}_{j}^{U}=\mathbf{e}^{i \omega h_{j} \mathbf{S}_{3}^{U}}$ representa a matriz propagadora descendente e ascendente, respectivamente, na $j$-ésima camada.

A expressão acima traduz todo o processo de propagação da onda transmitida através da camada e 0 operador

$$
(\mathbf{I}-\boldsymbol{\Pi})^{-1}=\mathbf{I}+\boldsymbol{\Pi}+\boldsymbol{\Pi}^{2}+\boldsymbol{\Pi}^{3}+\cdots
$$

constitui o chamado operador de reverberação (Protázio, 1994) e cada um de seus termos é responsável pela descrição de cada múltipla envolvida na propagação através da camada.

Seguindo o raciocínio anterior, o coeficiente $\mathbf{r}$ pode ser calculado como:

$$
\mathbf{r}=\left\{\mathbf{R}_{01}+\mathbf{T}_{10} \boldsymbol{\Lambda}_{1}^{U} \mathbf{R}_{12}(\mathbf{I}-\boldsymbol{\Pi})^{-1} \boldsymbol{\Lambda}_{1}^{D} \mathbf{T}_{01}\right\} \mathbf{i} .
$$

\section{EXPERIMENTOS NUMÉRICOS}

Para mostrar a robustez do modelo apresentado, experimentos numéricos na forma de sismogramas sintéticos de ondas planas e hodogramas são apresentados.

Os sismogramas são apresentados no domínio $(t, s)$, sendo

$$
s=\sqrt{s_{1}^{2}+s_{2}^{2}},
$$

com $s_{1}=\sin \theta \cos \phi / \alpha, s_{2}=\sin \theta \sin \phi / \alpha$ e $\alpha$, a velocidade longitudinal do meio incidente projetada no plano sagital. A assinatura no tempo, $t$, do pulso incidente é dada pela função: $f(t)=\sin \left(\omega_{c} t\right)$, sendo $\omega_{c}=25 \mathrm{~Hz}$ a frequência do pulso. Os ângulos de incidência ( $\theta$ ) foram calculados no intervalo [ $0^{\circ}$, $90^{\circ}$ ], 0 ângulo azimutal foi de $\phi=0^{\circ}$ no primeiro experimento e $\phi=75^{\circ}$ no segundo. Uma observação geral com relação aos sismogramas sintéticos das Figuras 3a, 3b, 5a e 5b é 0 fato da escala do tempo estar invertida, pois os cálculos do tempo são dependentes da vagarosidade.

Além dos sismogramas foram também calculados os hodogramas para ângulos de incidência de dez em dez graus entre 0 e $90^{\circ}$.

\section{Experimento isotrópico}

No primeiro experimento, os sismogramas foram calculados para um modelo litológico de uma interface plano-horizontal que separam dois semiespaços, sendo: (i) o superior isotrópico (folhelho) com densidade, velocidades compressional e cisalhante dadas por $\rho_{1}=2,35 \mathrm{~km} / \mathrm{s}, \alpha_{1}=3,3 \mathrm{~km} / \mathrm{s}$ e $\beta_{1}=1,7 \mathrm{~km} / \mathrm{s}$, respectivamente; (ii) 0 inferior isotrópico (arenito) com densidade, velocidade compressional e cisalhante dadas por $\rho_{2}=2,49 \mathrm{~km} / \mathrm{s}$, $\alpha_{2}=4,2 \mathrm{~km} / \mathrm{s}$ e $\beta_{2}=2,7 \mathrm{~km} / \mathrm{s}$, respectivamente.

Quanto à reflexão da onda $P$ na interface observa-se nos sismogramas da Figura 3a que a partir do ângulo crítico de propagação ocorre um aumento significativo da amplitude do pulso. Já a transmissão da onda $P$ na interface (Fig. 3b) se estende somente até 0 valor crítico de $s$.

Todos os sismogramas foram projetados no plano de incidência o que explica a ausência de evento refletido e transmitido na segunda componente no experimento isotrópico.

Nos hodogramas da reflexão (Fig. 4a) observa-se que são lineares, a variação da direção de propagação acompanha a direção de fase e a energia aumenta significantemente a partir do ângulo crítico. Já os hodogramas da transmissão (Fig. 4b) apresentam polarização linear até o ponto crítico de incidência e a partir deste, polarização elíptica. Esta é uma característica marcante neste tipo de experimento.

\section{Experimento anisotrópico}

Para o segundo experimento, visando constatar os efeitos da anisotropia no modelo, considerou-se o mesmo modelo anterior só que o semiespaço subjacente foi fraturado (Hudson, 1981) com densidade de fratura de $\varepsilon=0,1$, razão entre eixos $D=$ 0,0001, tornando-se um anisotrópico com matriz de parâmetros elásticos:

$$
C=\left[\begin{array}{cccccc}
26,1819 & 4,5418 & 4,5418 & 0 & 0 & 0 \\
4,5418 & 43,3897 & 7,0855 & 0 & 0 & 0 \\
4,5418 & 7,0855 & 43,3897 & 0 & 0 & 0 \\
0 & 0 & 0 & 18,1521 & 0 & 0 \\
0 & 0 & 0 & 0 & 14,2555 & 0 \\
0 & 0 & 0 & 0 & 0 & 14,2555
\end{array}\right]
$$

Quanto à reflexão da onda $P$ na interface (Fig. 5a), como era de se esperar, possui as mesmas características do experimento isotrópico. 
(a)
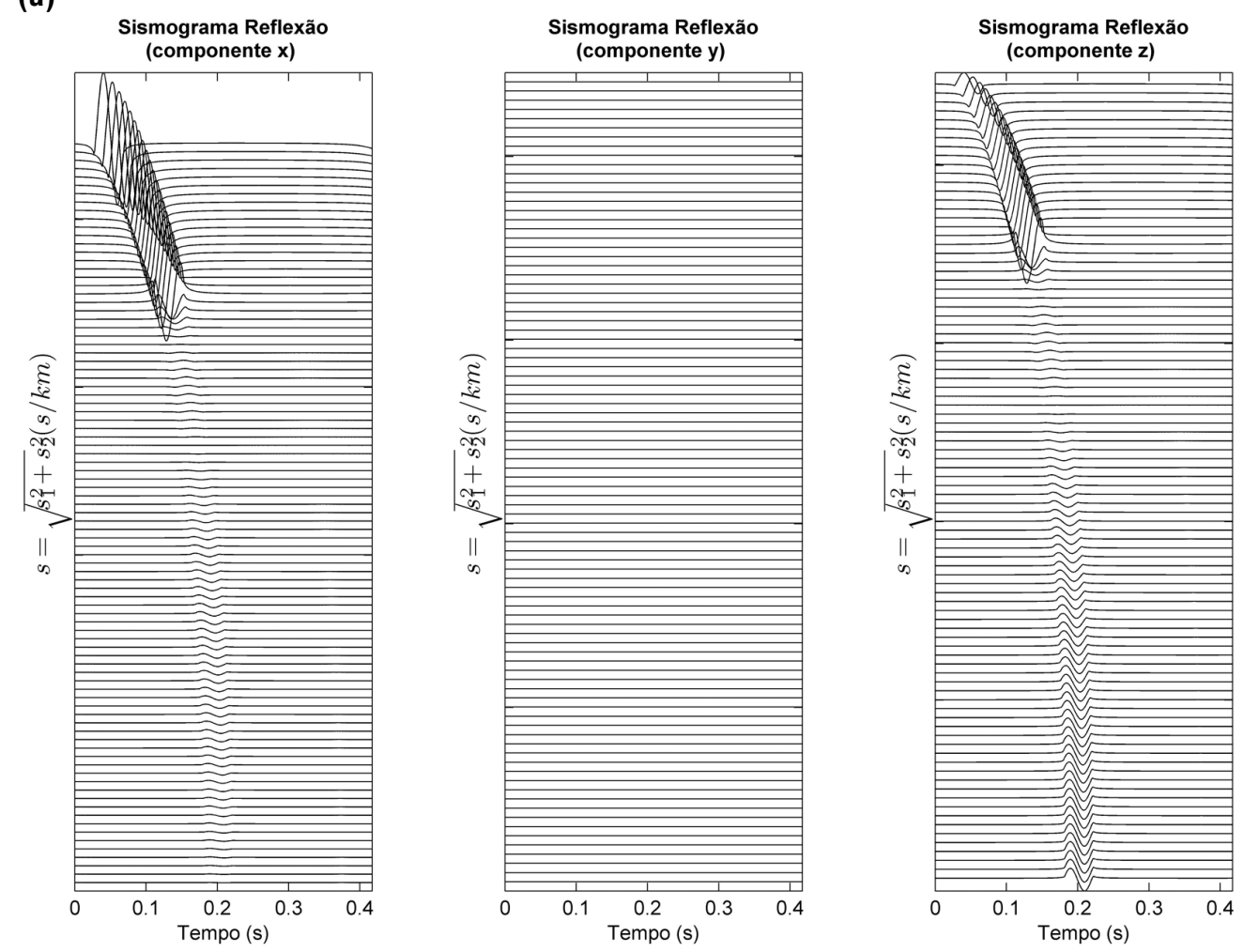

(b)
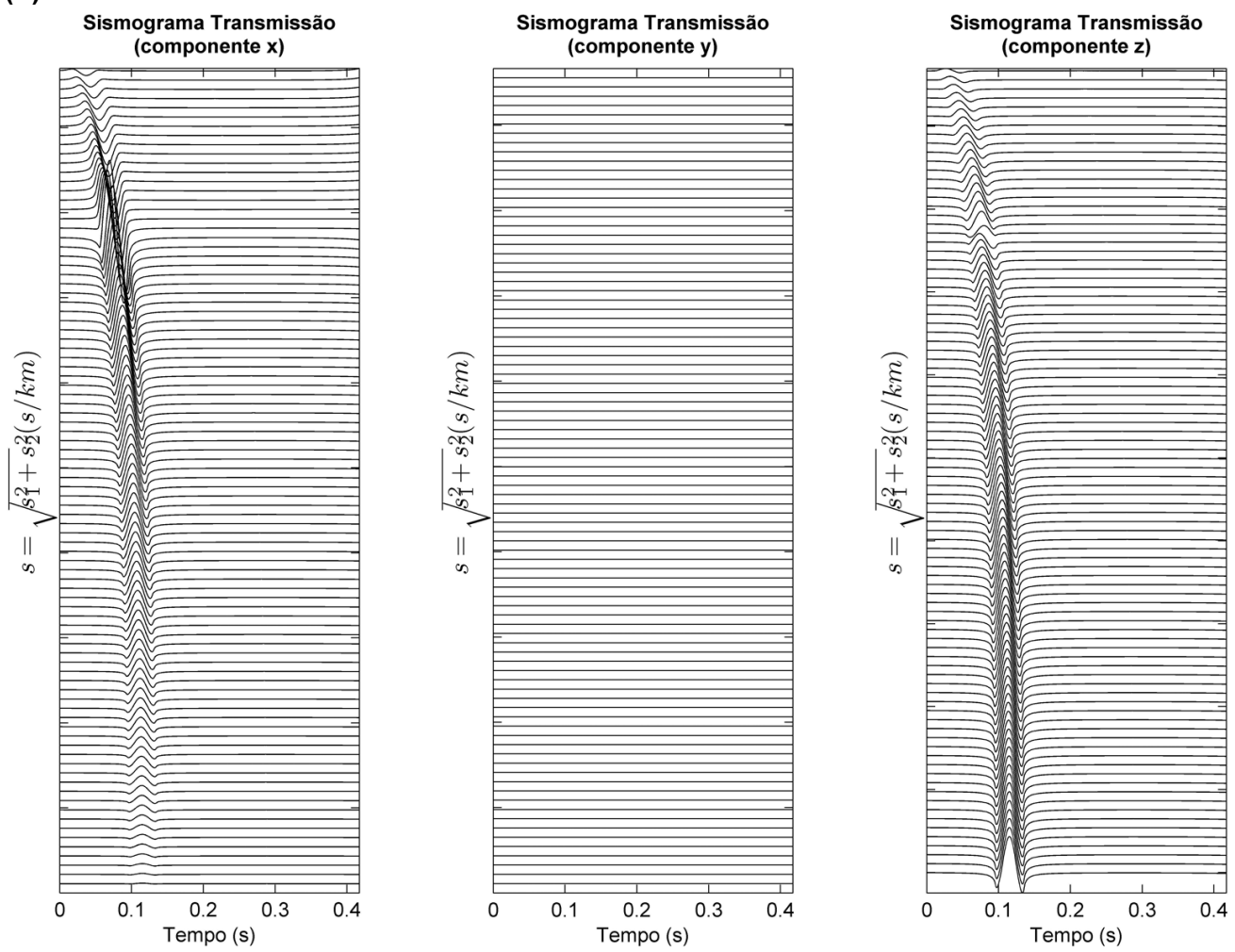

Figura 3 - Sismogramas sintéticos para reflexão (a) e transmissão (b) no modelo litológico isotrópico-isotrópico do primeiro experimento. 
(a)

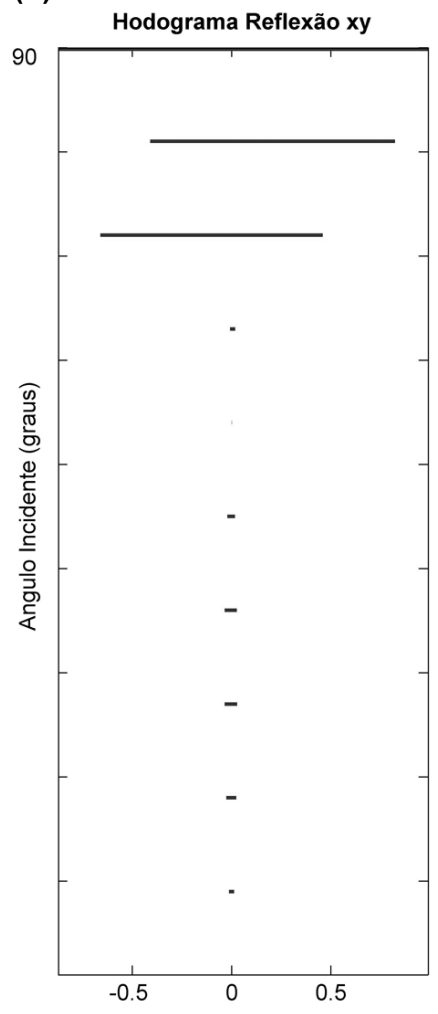

(b)

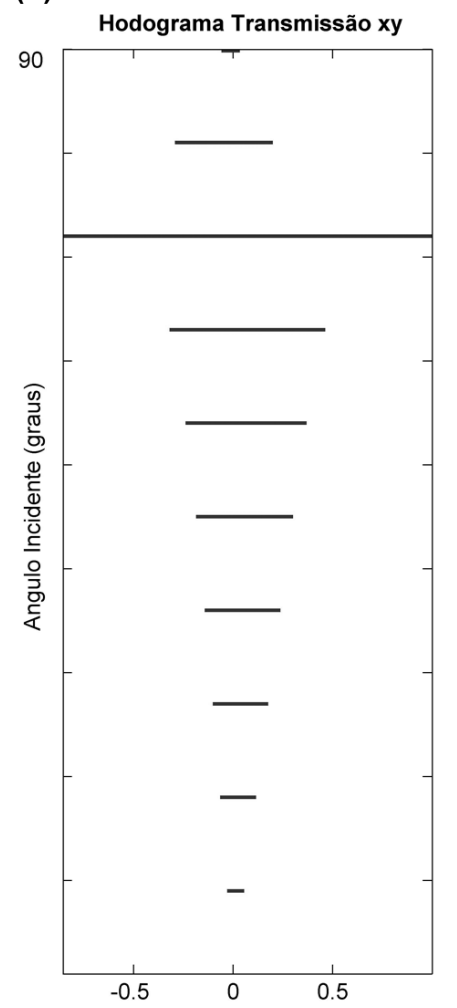

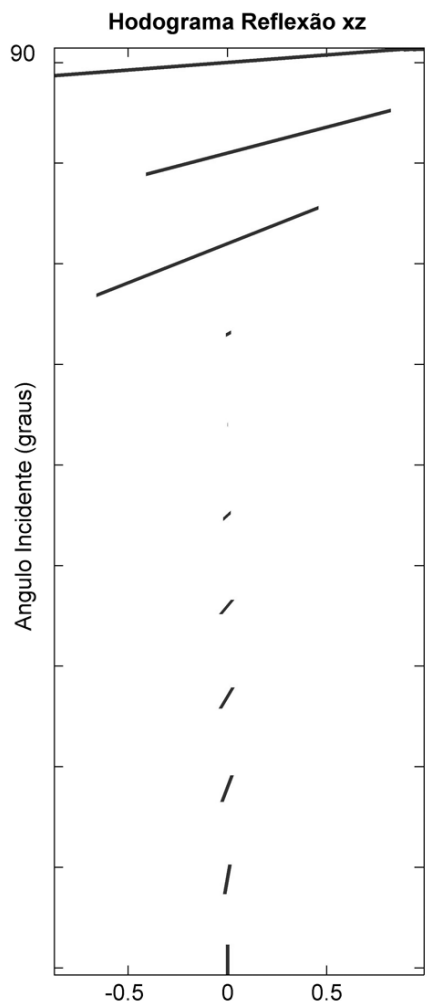
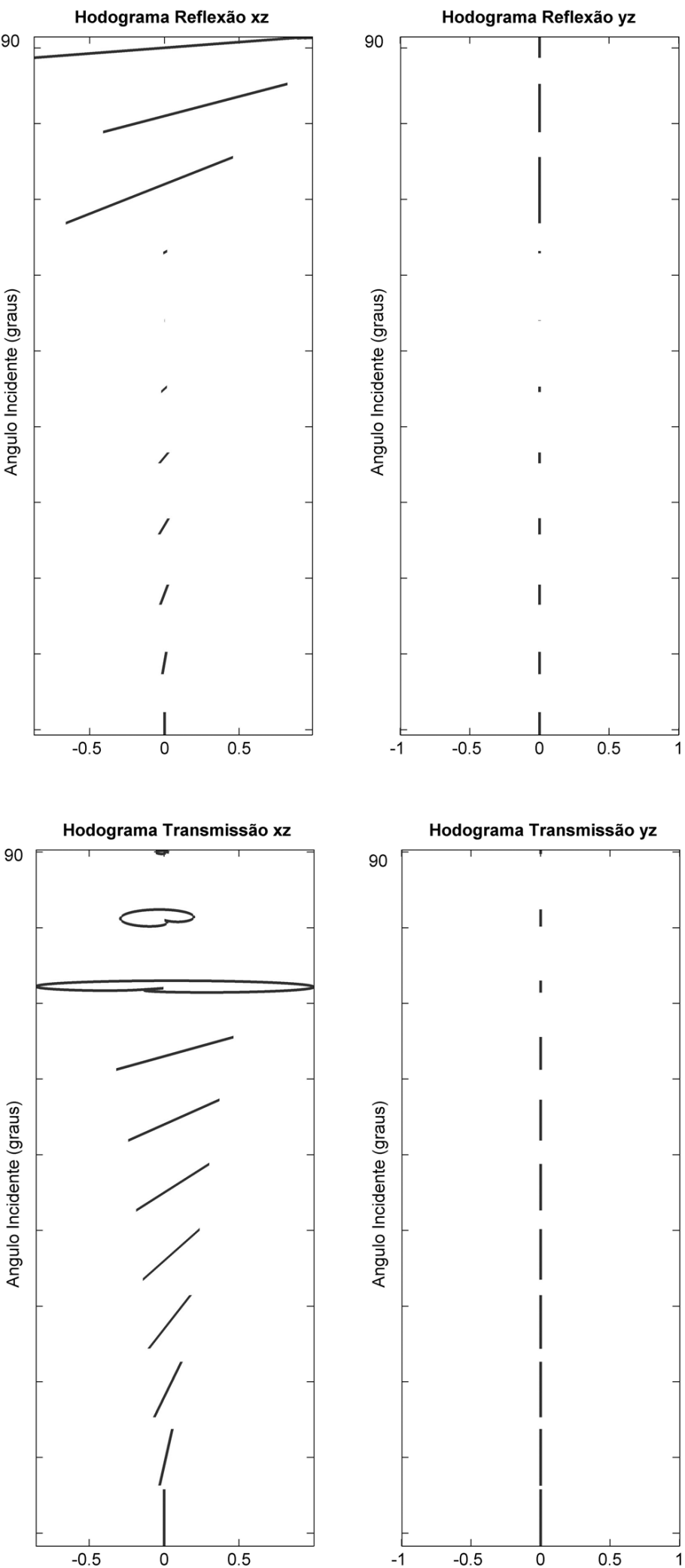

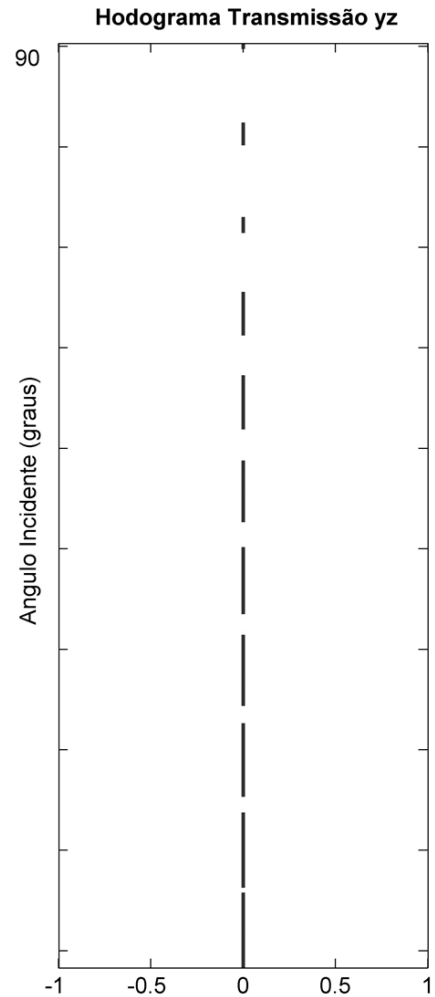

Figura 4 - Hodogramas para reflexão (a) e transmissão (b) no modelo litológico isotrópico-isotrópico do primeiro experimento. 
(a)
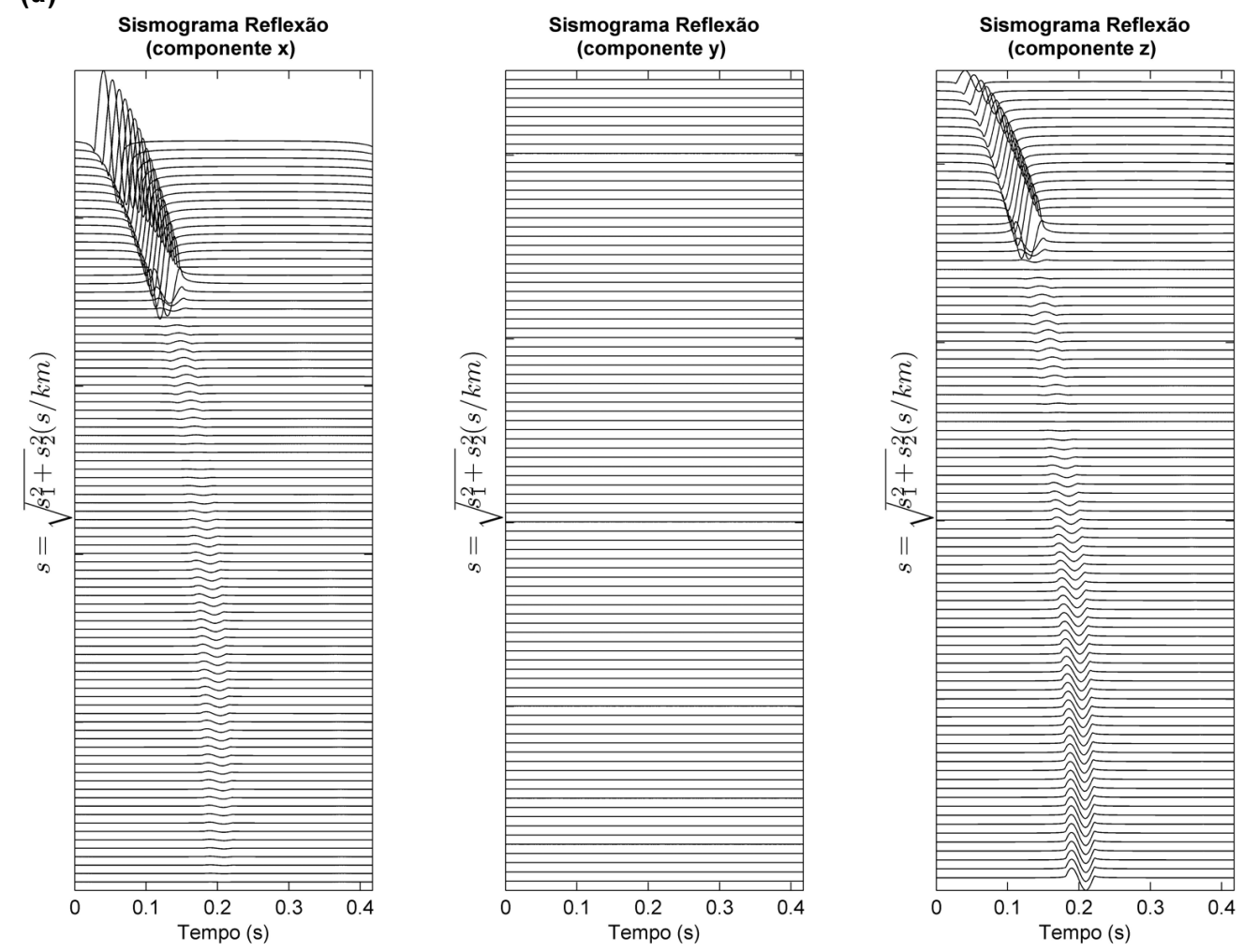

(b)
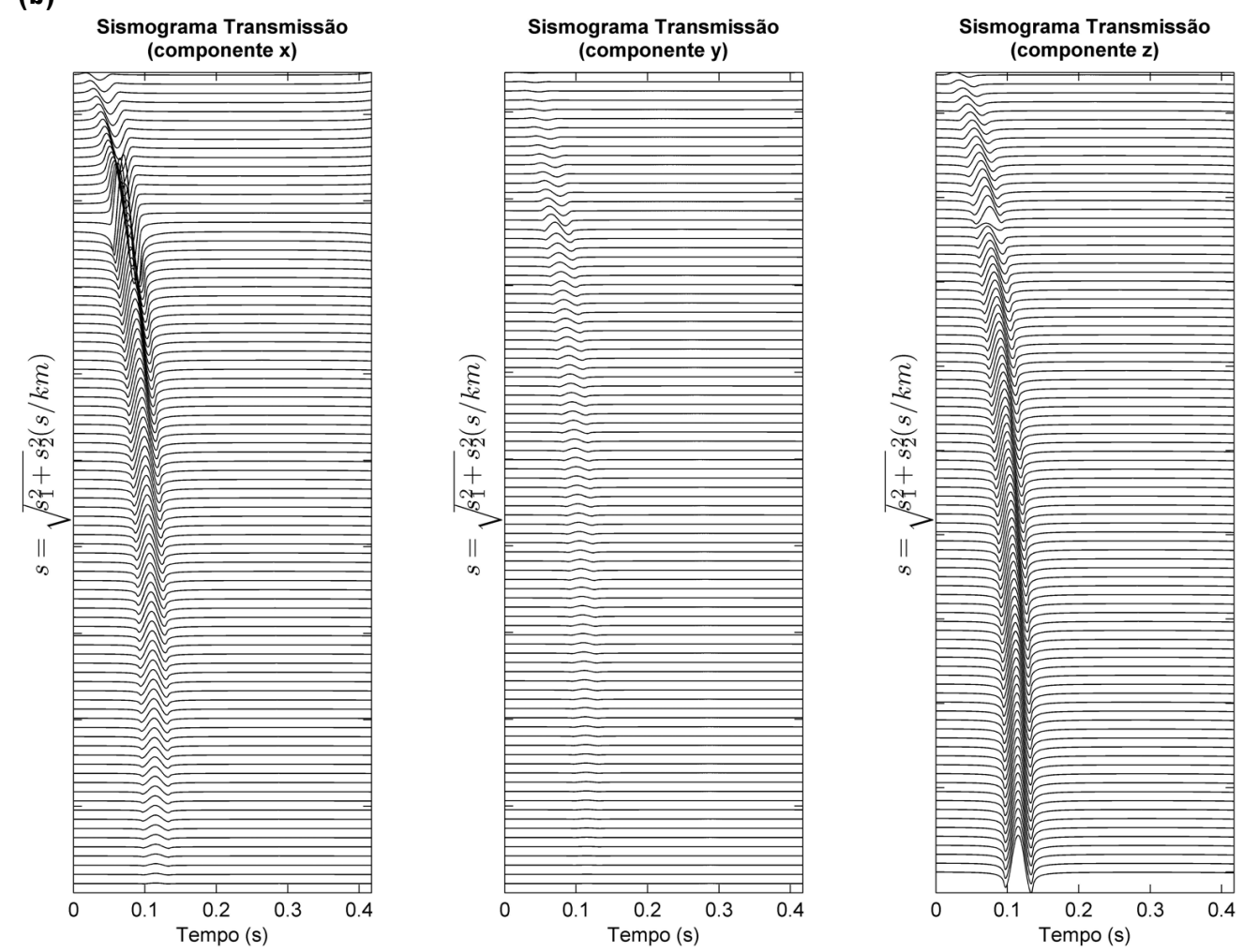

Figura 5 - Sismogramas sintéticos para reflexão (a) e transmissão (b) no modelo litológico isotrópico-anisotrópico do segundo experimento. 
(a)

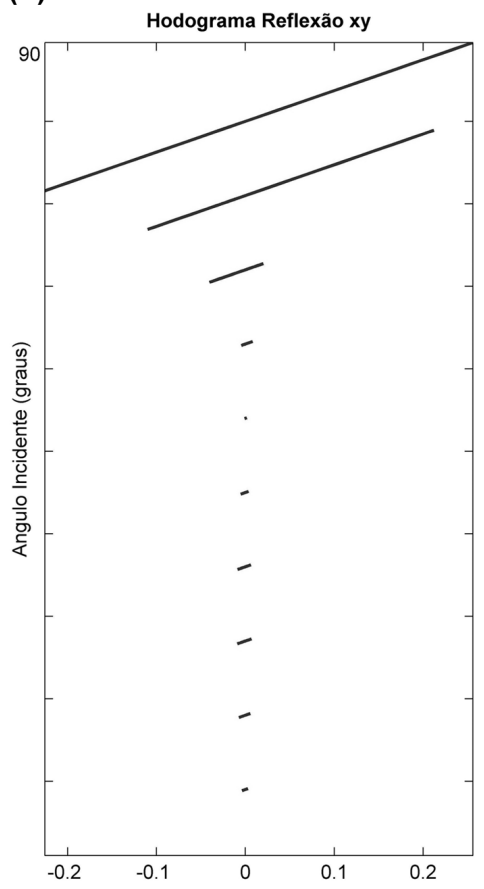

(b)

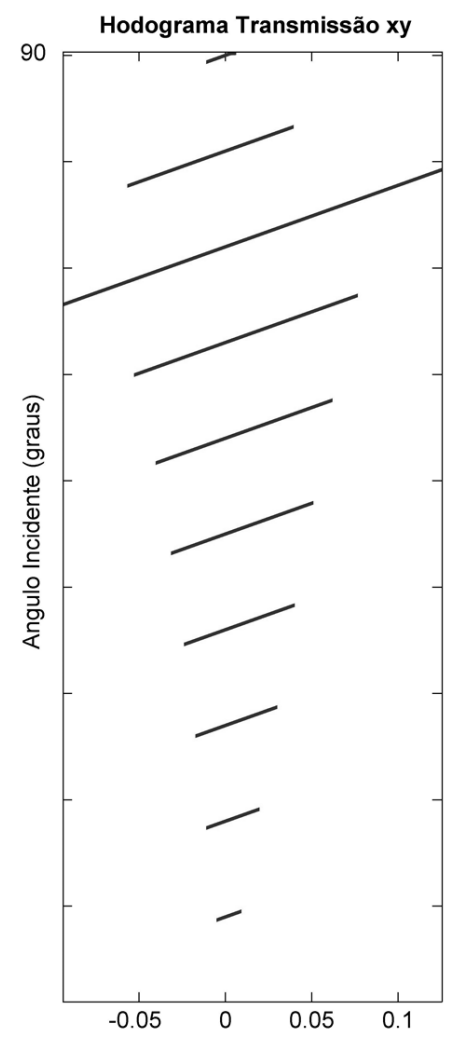

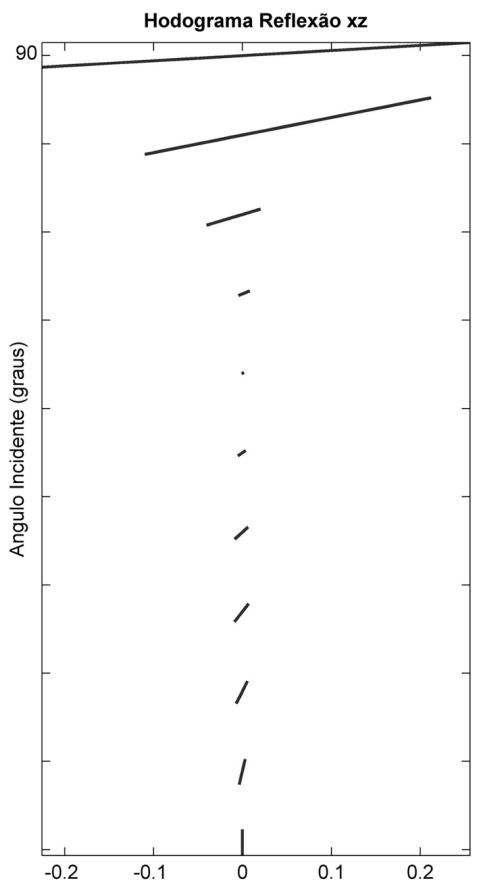
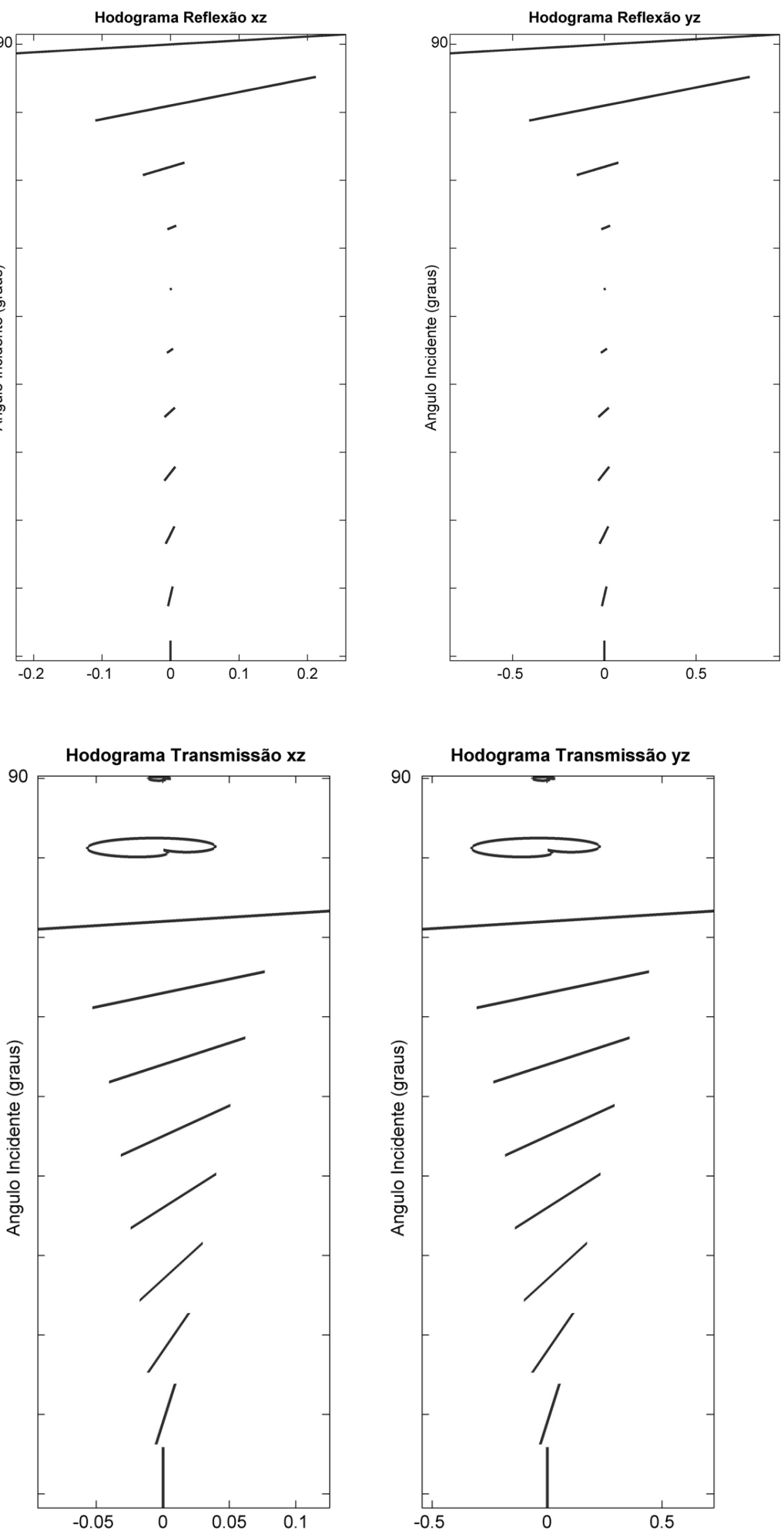

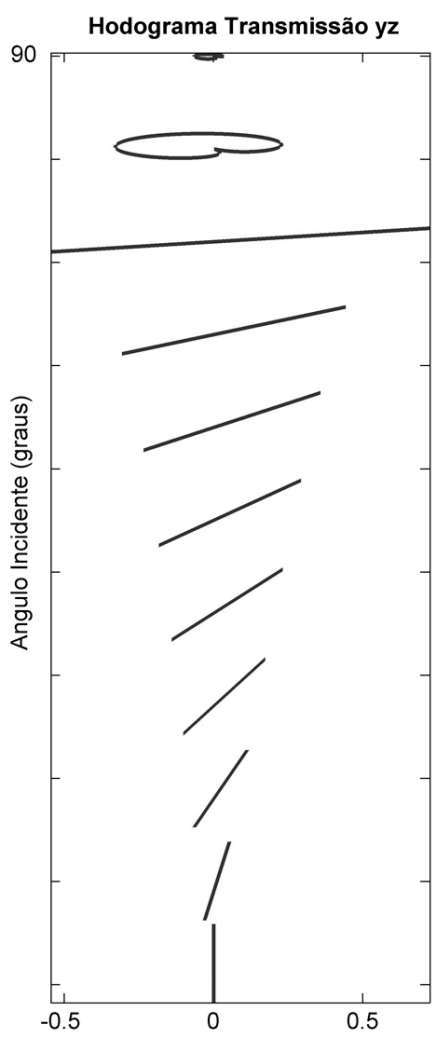

Figura 6 - Hodogramas para reflexão (a) e transmissão (b) no modelo litológico isotrópico-anisotrópico do segundo experimento. 
Na transmissão (Fig. 4b) os sismogramas apresentam eventos na segunda componente, que é característica marcante em meios anisotrópicos.

Para os hodogramas da Figura 6a observa-se o mesmo padrão do experimento isotrópico, mas com uma perda de energia próximo ao ponto crítico.

Já os hodogramas da Figura 6b apresentam também, o mesmo padrão do experimento isotrópico, mas a mudança do padrão elíptico acusa variação no ponto crítico o que também é marcante em experimentos considerando a presença de anisotropia.

\section{CONCLUSÃO}

As conclusões e potencialidades deste trabalho para a exploração sísmica são as seguintes:

1. Foi apresentado um formalismo consistente para se modelar 0 espalhamento de ondas em meios anisotrópicos gerais através da obtenção explícita dos campos de deformação e tração como função das matrizes propagadoras, de polarização e impedância. Desse modo, foi possível obter os coeficientes de reflexão e transmissão do espalhamento através de uma interface plana horizontal separando meios anisotrópicos e, posteriormente, generalizar para várias camadas. Ao formalismo acima descrito introduziuse um sinal analítico para se estudar o espalhamento de pulsos em meios anisotrópicos gerais.

2. Para verificar a consistência do formalismo acima descrito foram apresentados experimentos numéricos na forma de sismogramas sintéticos de ondas planas e hodogramas para se estudar a reflexão e a transmissão de ondas $P$ em interface plana separando meios isotrópicos e, posteriormente, meios isotrópico-anisotrópico (isotrópico fraturado) para confirmar a consistência da metodologia se comparado a estudos consagrados na literatura.

\section{AGRADECIMENTOS}

0 primeiro autor agradece ao CNPa pela concessão de Bolsa de Doutorado.

\section{REFERÊNCIAS}

AKI K \& RICHARD PG. 1980. Quantitative seismology. W.H. Freeman \& Co., N.Y., v.2, $557 \mathrm{p}$.

CRAMPIN S. 1985. Evaluation of anisotropy by shear-wave splitting. Geophysics, 50(1): 142-152.

FRYER GJ \& FRAZER LN. 1987. Seismic waves in stratified anisotropic media - II: elastodynamic eigensolutions for some anisotropic systems. Geophys. J. Roy. Astron. Soc., 91: 73-101.

GOMES ENS. 1999. Refletividade de ondas $P$ em meios anisotrópicos. (Dissertação de Mestrado), Universidade Federal do Pará, Centro de Ciências Exatas e Naturais, Departamento de Matemática, Belém, 102 p.

HUDSON JA. 1981. Wave speeds and attenuation of elastic waves in material containing cracks. Geophys. J. Roy. Astron. Soc., 64: 133150.

MALLICK S \& FRAZER LN. 1988. Rapid computation of multioffset vertical seismic profile synthetic seismograms for layered media. Geophysics, 53(4): 479-491.

PEREIRA RM. 2003. Propagação de Pulsos em Meios Anisotrópicos. (Dissertação de Mestrado), Universidade Federal do Pará, Centro de Geociências, Pós-Graduação em Geofísica, Belém, 66 p.

PROTÁZIO JS. 1994. Espalhamento elástico em meios anisotrópicos estratificados. (Tese de Doutorado), Universidade Federal do Pará, Centro de Geociências, Pós-Graduação em Geofísica, Belém, 262 p.

WOODHOUSE JH. 1974. Surface waves in a laterally varying layered structure. Geophys. J. Roy. Astron. Soc., 37: 461-490.

\section{NOTAS SOBRE OS AUTORES}

Rubenvaldo Monteiro Pereira é formado em Matemática (UFPa/1999). Mestre em Geofísica (UFPa/2004). Foi professor substituto no Departamento de Matemática da Universidade Federal do Pará (2001-2003). Atualmente é professor assistente da Faculdade de Matemática da UFPa-Campus Tocantins Cametá. Áreas de interesse: matemática aplicada, sísmica e bioestatística.

João dos Santos Protázio é formado em Matemática (UFPa/1972). Mestre em Matemática (PUC-RJ/1976). Doutor em Geofísica (UFPa/1994). Estágio de pósdoutoramento no IMECC/IG/UNICAMP (1995). Foi pesquisador no Laboratório Nacional de Computação Científica-LNCC (1985-1987). Foi professor auxiliar na Pontifícia Universidade Católica do Rio de Janeiro - PUC (1973-1979). Foi professor da Universidade Federal do Pará (1980-2004). Foi professor associado dos Programas de Pós-Graduação em Matemática e Estatística/UFPa e CPGf/CG/UFPa. Atualmente é professor da Faculdade de Estatística do ICEN/UFPa. Áreas de interesse: matemática aplicada, sísmica e bioestatística.

Jessé Carvalho Costa é formado em Física (UFPa/1983). Mestre e Doutor em Geofísica (UFPa/1987, 1993 respectivamente). Summer Student na Schlumberger Cambridge Research em 1991 e 1992. Estágio de pós-doutoramento no Departamento de Geofísica da Universidade de Stanford (1994,1996) e Visiting Assistent Professor no Departamento de Geofísica da Universidade de Stanford (1995). Foi professor da Universidade Federal do Pará no Departamento de Física (1989-2003). Atualmente é professor do PPGG do Instituto de Geociências/UFPa. Áreas de interesse: modelamento e imageamento sísmico e tomografia. 